\title{
Some Identities on the Generalized Changhee-Genocchi Polynomials and Numbers
}

\author{
Dan-Dan Zhao* and Wuyungaowa \\ Department of Mathematics, College of Sciences and Technology, Inner Mongolia University, Huhhot, China \\ Email: zhaodandan1231@163.com
}

\begin{abstract}
In this paper, we generalize the generating function of the Changhee-Genocchi polynomials. In particular, by means of the method of generating functions and Riordan arrays, we study some properties of the generalized Changhee-Genocchi polynomials. At the same time, we establish some identities between the generalized Changhee-Genocchi polynomials and other combinatorial sequences.
\end{abstract}

Keywords: Generalized Changhee-Genocchi polynomials, generalized Changhee-Genocchi numbers, generating functions, Riordan arrays.

\section{Introduction}

In 2016, Byung-Moon Kim first introduced the concept of Changhee-Genocchi polynomials, the ChangheeGenocchi polynomials are defined by the generating function (see[1])

$$
\sum_{n=0}^{\infty} C G_{n}(x) \frac{t^{n}}{n !}=\frac{2 \log (1+t)}{2+t}(1+t)^{x}
$$

When $x=0, C G_{n}=C G_{n}(0)$ are called the Changhee-Genocchi numbers.

In addition, Byung-Moon Kim also gived the Changhee-Genocchi polynomials of the order $r$ by the generating function (see[1])

$$
\sum_{n=0}^{\infty} C G_{n}^{(r)}(x) \frac{t^{n}}{n !}=\left(\frac{2 \log (1+t)}{2+t}\right)^{r}(1+t)^{x} .
$$

For convenience, let us recall some definitions and notations. Here, the generalized Harmonic numbers are defined by the generating function (see[2])

$$
\sum_{n=0}^{\infty} H_{n, k, r}(\alpha, \beta) t^{n}=\frac{(-\log (1-\alpha t))^{r}}{(1-\beta t)^{k}} .
$$

As is well known, the higher-order Changhee numbers are defined by the generating function (see[3])

$$
\sum_{n=0}^{\infty} C h_{n}^{(k)} \frac{t^{n}}{n !}=\left(\frac{2}{2+t}\right)^{k} .
$$

We consider the $\mathrm{n}$-th twisted Daehee polynomials of order $\mathrm{k}$, which are defined by the generating function (see[4])

$$
\sum_{n=0}^{\infty} D_{n, \xi}^{(k)}(x) \frac{t^{n}}{n !}=\left(\frac{\log (1+\xi t)}{\xi t}\right)^{k}(1+\xi t)^{x}
$$

In special case, when $x=0, D_{n, \xi}^{(k)}(0)=D_{n, \xi}^{(k)}$ are called twisted Daehee numbers of order r. When $\xi=1$, $D_{n, 1}^{(k)}=D_{n}^{(k)}$ are higher-order Daehee numbers. 
Next, we give several kinds of generating functions which we need in this paper(see[5,6,7,8,9])

$$
\begin{aligned}
& \sum_{n=0}^{\infty} G_{n}^{(r)} \frac{t^{n}}{n !}=\left(\frac{2 t}{e^{t}+1}\right)^{r} . \\
& \sum_{n=0}^{\infty} B_{n}^{(r)} \frac{t^{n}}{n !}=\left(\frac{t}{e^{t}-1}\right)^{r} . \\
& \sum_{n=0}^{\infty} b_{n}^{(r)} \frac{t^{n}}{n !}=\left(\frac{t}{\log (1+t)}\right)^{r} . \\
& \sum_{n=0}^{\infty} \frac{G_{n}^{(x)}}{2^{n}} \frac{t^{n}}{n !}=\left(\frac{2}{e^{t}+1}\right)^{x} .
\end{aligned}
$$

Let $\Im=\Re[[t]]$ be the ring of the formal power series with real coefficients in some indeterminate $t$, if $f(t) \in \Im$ and $f(t)=\sum_{n=0}^{\infty} f_{n} t^{n}$, let $\left[t^{n}\right] f(t)$ be the coefficient of $\left[t^{n}\right]$ in the formal power series of $f(t)$. If $f(t)$ and $g(t)$ are formal power series, then we get the following relations:

$$
\begin{gathered}
{\left[t^{n}\right](\alpha f(t)+\beta g(t))=\alpha\left[t^{n}\right] f(t)+\beta\left[t^{n}\right] g(t) .} \\
\sum_{j=0}^{n}\left[t^{j}\right] f(t)\left[t^{n-j}\right] g(t)=\left[t^{n}\right] f(t) g(t) .
\end{gathered}
$$

A Riordan array is a couple $D=(d(t), h(t))$ in which $d(t), h(t) \in \Im$ and $h_{0}=h(0)=0$. It defines an infinite lower triangular array $\left(d_{n, k}\right)_{n, k \in N}$ according to the rule $d_{n, k}=\left[t^{n}\right] d(t) h(t)^{k}$. So we set $\left\{d_{n, k}\right\}=$ $(d(t), h(t))$. Let $D=(d(t), h(t))$ be a Riordan array and $f(t)$ be the generating function of the sequence $\left\{f_{i}\right\}_{i \in N}$, we have (see[10])

$$
\sum_{k=0}^{\infty} d_{n, k} f_{k}=\left[t^{n}\right] d(t) f(h(t))=\left[t^{n}\right] d(t)\left[\left.f(y)\right|_{y=h(t)}\right] .
$$

Recently, many papers have been devoted to the study of the Changhee-Genocchi polynomials and numbers by various methods. In this paper, we generalize the generating function of the ChangheeGenocchi polynomials on the basis of these papers, and investigate some interesting identities related to the generalized Changhee-Genocchi polynomials and numbers.

\section{Some Properties of the Generalized Changhee-Genocchi Polynomials}

In this paper, we consider the generalized Changhee-Genocchi polynomials which are defined by the generating function

$$
\sum_{n=0}^{\infty} C G_{n}^{k, r}(\alpha, \beta \mid x) \frac{t^{n}}{n !}=\frac{2^{k} \log ^{r}(1+\alpha t)}{(2+\beta t)^{k}}(1+\beta t)^{\alpha x}
$$

where $k \geq 1, r \geq 1$ are intergers, $\alpha$ and $\beta$ are real numbers, and $\alpha \beta \neq 0$.

When $x=0, C G_{n}^{k, r}(\alpha, \beta \mid 0)=C G_{n}^{k, r}(\alpha, \beta)$ are called the generalized Changhee-Genocchi numbers.

Particularly, when $\alpha=\beta=1$ and $k=r, C G_{n}^{r, r}(1,1 \mid x)=C G_{n}^{(r)}(x), n \geq 0$. 
When $\alpha=\beta=1$ and $k=r=1, C G_{n}^{1,1}(1,1 \mid x)=C G_{n}(x), n \geq 0$.

In this section, we study some properties of the generalized Changhee-Genocchi polynomials by the generation function method.

For equation (13), we also get

$$
\sum_{n=0}^{\infty} C G_{n}^{k, r}(\alpha, \beta \mid x) \frac{t^{n}}{n !}=\frac{2^{k} \log ^{r}(1+\alpha t)}{[1+(1+\beta t)]^{k}}(1+\beta t)^{\alpha x} .
$$

Hence, we have

$$
\begin{aligned}
2^{k} \log ^{r}(1+\alpha t) & =[1+(1+\beta t)]^{k} \sum_{n=0}^{\infty} C G_{n}^{k, r}(\alpha, \beta \mid x) \frac{t^{n}}{n !}(1+\beta t)^{-\alpha x} \\
& =\sum_{l=0}^{k}\left(\begin{array}{c}
k \\
l
\end{array}\right)(1+\beta t)^{l-\alpha x} \sum_{n=0}^{\infty} C G_{n}^{k, r}(\alpha, \beta \mid x) \frac{t^{n}}{n !} \\
& =\sum_{i=0}^{\infty} \sum_{l=0}^{k}\left(\begin{array}{c}
k \\
l
\end{array}\right)(l-\alpha x)_{i} \beta^{i} \frac{t^{i}}{i !} \sum_{n=0}^{\infty} C G_{n}^{k, r}(\alpha, \beta \mid x) \frac{t^{n}}{n !} \\
& =\sum_{n=0}^{\infty} \sum_{m=0}^{n} \sum_{l=0}^{k}\left(\begin{array}{c}
k \\
l
\end{array}\right)\left(\begin{array}{c}
l-\alpha x \\
m
\end{array}\right) \frac{\beta^{m}}{(n-m) !} C G_{n-m}^{k, r}(\alpha, \beta \mid x) t^{n} .
\end{aligned}
$$

On the other hand, we also can get

$$
\begin{aligned}
2^{k} \log ^{r}(1+\alpha t) & =[1+(1+\beta t)]^{k} \sum_{n=0}^{\infty} C G_{n}^{k, r}(\alpha, \beta \mid x) \frac{t^{n}}{n !}(1+\beta t)^{-\alpha x} \\
& =\sum_{l=0}^{k}\left(\begin{array}{c}
k \\
l
\end{array}\right)(1+\beta t)^{l} \sum_{n=0}^{\infty} C G_{n}^{k, r}(\alpha, \beta \mid x) \frac{t^{n}}{n !} \sum_{n=0}^{\infty}\left(\begin{array}{c}
-\alpha x \\
n
\end{array}\right)(-\beta)^{n} t^{n} \\
& =\sum_{n=0}^{\infty} \sum_{m=0}^{n} \sum_{l=m}^{k}\left(\begin{array}{c}
k \\
l
\end{array}\right)\left(\begin{array}{c}
l \\
m
\end{array}\right)\left(\begin{array}{c}
-\alpha x \\
n-m
\end{array}\right)(-1)^{n-m} \beta^{n} t^{n} \sum_{n=0}^{\infty} C G_{n}^{k, r}(\alpha, \beta \mid x) \frac{t^{n}}{n !} \\
& =\sum_{n=0}^{\infty} \sum_{p=0}^{n} \sum_{m=0}^{p} \sum_{l=m}^{k}\left(\begin{array}{c}
k \\
l
\end{array}\right)\left(\begin{array}{c}
l \\
m
\end{array}\right)\left(\begin{array}{c}
-\alpha x \\
p-m
\end{array}\right) C G_{n-p}^{k, r}(\alpha, \beta \mid x) \frac{(-1)^{p-m} \beta^{p}}{(n-p) !} t^{n} .
\end{aligned}
$$

Theorem 1. Let $n$ be nonnegative integers, $k \geq 1$ and $r \geq 1$ are integers, we have

$$
\begin{aligned}
& \sum_{p=0}^{n} \sum_{m=0}^{p} \sum_{l=m}^{k}\left(\begin{array}{c}
k \\
l
\end{array}\right)\left(\begin{array}{c}
l \\
m
\end{array}\right)\left(\begin{array}{c}
-\alpha x \\
p-m
\end{array}\right) C G_{n-p}^{k, r}(\alpha, \beta \mid x) \frac{(-1)^{p-m} \beta^{p}}{(n-p) !} \\
& =\sum_{m=0}^{n} \sum_{l=0}^{k}\left(\begin{array}{c}
k \\
l
\end{array}\right)\left(\begin{array}{c}
l-\alpha x \\
m
\end{array}\right) C G_{n-m}^{k, r}(\alpha, \beta \mid x) \frac{\beta^{m}}{(n-m) !} \\
& =2^{k} r ! \alpha^{n} \frac{B_{n, r}(1,-1,2 !,-3 !, \ldots)}{n !} .
\end{aligned}
$$

Proof By comparing the coefficients of $t^{n}$ on both sides of the equation (14) and (15), theorem 1 is proved.

Theorem 2. Let $n$ be nonnegative integers, $k, r, p, q \geq 1$ are integers, we have

$$
\sum_{j=0}^{n} \sum_{m=0}^{j} \sum_{l=0}^{k}\left(\begin{array}{c}
k \\
l
\end{array}\right)\left(\begin{array}{c}
l-\alpha x \\
m
\end{array}\right) \frac{C G_{j-m}^{k, r}(\alpha, \beta \mid x)}{(j-m) !} \frac{C G_{n-j}^{p, q}(\alpha, \beta \mid y)}{(n-j) !} n ! \beta^{m}=2^{k} C G_{n}^{p, q+r}(\alpha, \beta \mid y) .
$$


Proof By equation (9) and equation (13), we get

$$
\begin{aligned}
& \sum_{j=0}^{n} \sum_{m=0}^{j} \sum_{l=0}^{k}\left(\begin{array}{c}
k \\
l
\end{array}\right)\left(\begin{array}{c}
l-\alpha x \\
m
\end{array}\right) \frac{\beta^{m}}{(j-m) !} C G_{j-m}^{k, r}(\alpha, \beta \mid x) \frac{C G_{n-j}^{p, q}(\alpha, \beta \mid y)}{(n-j) !} \\
& =\sum_{j=0}^{n}\left[t^{j}\right] 2^{k} \log ^{r}(1+\alpha t)\left[t^{n-j}\right] \frac{2^{p} \log ^{q}(1+\alpha t)}{(2+\beta t)^{p}}(1+\beta t)^{\alpha y} \\
& =\left[t^{n}\right] 2^{k} \frac{2^{p} \log ^{r+q}(1+\alpha t)}{(2+\beta t)^{p}}(1+\beta t)^{\alpha y}=\frac{2^{k}}{n !} C G_{n}^{p, r+q}(\alpha, \beta \mid y) .
\end{aligned}
$$

Theorem 3. Let $n$ be nonnegative integers, $k, r \geq 1$ are integers, we have

$$
\sum_{m=0}^{n}\left(\begin{array}{c}
k \\
m
\end{array}\right)\left(\frac{\beta}{2}\right)^{m} C G_{n-m}^{k, r}(\alpha, \beta) \frac{n !}{(n-m) !}=r ! \alpha^{n} S_{1}(n, r) .
$$

Proof By equation (13), when $x=0$, we have

$$
\sum_{n=0}^{\infty} C G_{n}^{k, r}(\alpha, \beta) \frac{t^{n}}{n !}=\frac{2^{k} \log ^{r}(1+\alpha t)}{(2+\beta t)^{k}}
$$

Hence, we can get

$$
\left(1+\frac{\beta}{2} t\right)^{k} \sum_{n=0}^{\infty} C G_{n}^{k, r}(\alpha, \beta) \frac{t^{n}}{n !}=\log ^{r}(1+\alpha t) .
$$

Here, we simpify the left side of this equation

$$
\begin{aligned}
& \left(1+\frac{\beta}{2} t\right)^{k} \sum_{n=0}^{\infty} C G_{n}^{k, r}(\alpha, \beta) \frac{t^{n}}{n !}=\sum_{l=0}^{\infty}\left(\begin{array}{l}
k \\
l
\end{array}\right)\left(\frac{\beta}{2}\right)^{l} t^{l} \sum_{n=0}^{\infty} C G_{n}^{k, r}(\alpha, \beta) \frac{t^{n}}{n !} \\
& =\sum_{n=0}^{\infty} \sum_{m=0}^{n}\left(\begin{array}{c}
k \\
m
\end{array}\right)\left(\frac{\beta}{2}\right)^{m} C G_{n-m}^{k, r}(\alpha, \beta) \frac{t^{n}}{(n-m) !} .
\end{aligned}
$$

For the right side, we have (see[11])

$$
\log ^{r}(1+\alpha t)=\sum_{n=r}^{\infty} r ! \alpha^{n} S_{1}(n, r) \frac{t^{n}}{n !}
$$

By comparing the coefficients of $\frac{t^{n}}{n !}$, theorem 3 is proved.

Corollary 1. In theorem 3 , when $k=1$ and $n \geq 1$, we get

$$
C G_{n}^{1, r}(\alpha, \beta)+\frac{n \beta}{2} C G_{n-1}^{1, r}(\alpha, \beta)=r ! \alpha^{n} S_{1}(n, r)
$$

Corollary 2. In theorem 3 , when $\alpha=\beta=1$, and $k=r=1$, we get theoerm 11 of the reference [1].

Theorem 4. Let $n$ be nonegative integers, $k \geq 1, r \geq 2$ are integers, we have

$$
\begin{aligned}
& C G_{n+1}^{k, r}(\alpha, \beta \mid x)+\frac{k \beta}{2} C G_{n}^{k+1, r}(\alpha, \beta \mid x) \\
& =\sum_{m=0}^{n}\left(\begin{array}{c}
n \\
m
\end{array}\right)\left[\frac{(n-m) !}{(-\alpha)^{m-n}} \alpha r C G_{m}^{k, r-1}(\alpha, \beta \mid x)+x \beta^{n-m+1}(\alpha)_{n-m+1} C G_{m}^{k, r}(\alpha, \beta \mid x-1)\right]
\end{aligned}
$$


Proof Let's take the derivative about $t$, on both sides of equation (13), we get

$$
\begin{aligned}
& \sum_{n=1}^{\infty} C G_{n}^{k, r}(\alpha, \beta \mid x) \frac{t^{n-1}}{(n-1) !} \\
& =\left[\frac{-k \beta}{2} \frac{2^{k+1} \log ^{r}(1+\alpha t)}{(2+\beta t)^{k+1}}+\frac{2^{k} \log ^{r-1}(1+\alpha t)}{(2+\beta t)^{k}} \frac{\alpha r}{1+\alpha t}\right](1+\beta t)^{\alpha x}+\frac{2^{k} \log ^{r}(1+\alpha t)}{(2+\beta t)^{k}} \alpha \beta x(1+\beta t)^{\alpha x-1} \\
& =\frac{-k \beta}{2} \sum_{n=0}^{\infty} C G_{n}^{k+1, r}(\alpha, \beta \mid x) \frac{t^{n}}{n !}+\alpha r \sum_{n=0}^{\infty} C G_{n}^{k, r-1}(\alpha, \beta \mid x) \frac{t^{n}}{n !} \sum_{n=0}^{\infty}(-\alpha)^{n} t^{n} \\
& +\alpha \beta x \sum_{n=0}^{\infty} C G_{n}^{k, r}(\alpha, \beta \mid x-1) \frac{t^{n}}{n !} \sum_{n=0}^{\infty}(\alpha-1)_{n} \beta^{n} \frac{t^{n}}{n !} \\
& =\frac{-k \beta}{2} \sum_{n=0}^{\infty} C G_{n}^{k+1, r}(\alpha, \beta \mid x) \frac{t^{n}}{n !}+\sum_{n=0}^{\infty} \sum_{m=0}^{n}\left(\begin{array}{c}
n \\
m
\end{array}\right)\left[(n-m) !(-1)^{n-m} \alpha^{n-m+1} r C G_{m}^{k, r-1}(\alpha, \beta \mid x)\right. \\
& \left.+x \beta^{n-m+1}(\alpha)_{n-m+1} C G_{m}^{k, r}(\alpha, \beta \mid x-1)\right] \frac{t^{n}}{n !} .
\end{aligned}
$$

By comparing the coefficients of $\frac{t^{n}}{n !}$ on both sides of this equation, theorem 4 is proved.

Theorem 5. Let $n$ be nonegative integers, $k, r \geq 1$ and $m, l \geq 1$ are integers, we have

$$
\begin{aligned}
& \sum_{p=0}^{n}\left(\begin{array}{l}
n \\
p
\end{array}\right) C G_{p}^{k, r}(\alpha, \beta \mid x) C h_{n-p}^{(m)}(\alpha y) \beta^{n-p}=C G_{n}^{k+m, r}(\alpha, \beta \mid x+y), \\
& \sum_{p=0}^{n}\left(\begin{array}{l}
n \\
p
\end{array}\right) C G_{p}^{k, r}(\alpha, \beta \mid x) C G_{n-p}^{m, l}(\alpha, \beta \mid y)=C G_{n}^{k+m, r+l}(\alpha, \beta \mid x+y) .
\end{aligned}
$$

Proof

$$
\begin{aligned}
& \sum_{n=0}^{\infty} C G_{n}^{k, r}(\alpha, \beta \mid x) \frac{t^{n}}{n !} \sum_{n=0}^{\infty} C h_{n}^{(m)}(\alpha y) \frac{(\beta t)^{n}}{n !}=\sum_{n=0}^{\infty} \sum_{p=0}^{n}\left(\begin{array}{c}
n \\
p
\end{array}\right) C G_{p}^{k, r}(\alpha, \beta) C h_{n-p}^{(m)}(\alpha y) \beta^{n-p} \frac{t^{n}}{n !} \\
& =\frac{2^{k} \log ^{r}(1+\alpha t)}{(2+\beta t)^{k}}\left(\frac{2}{2+\beta t}\right)^{m}(1+\beta t)^{\alpha(x+y)}=\sum_{n=0}^{\infty} C G_{n}^{k+m, r}(\alpha, \beta \mid x+y) \frac{t^{n}}{n !} .
\end{aligned}
$$

By comparing the coefficients of $\frac{t^{n}}{n !}$ on both sides of this equation, equation (20) is proved. The proof of (21) is similar to that of $(20)$.

\section{Identities Involving the Generalized Changhee-Genocchi Polynomials and Numbers}

In this section, we establish some identities which are related to the generalized Changhee-Genocchi polynomials. Then we find the Riordan array of the generalized Changhee-Genocchi numbers, and give several identities by means of the Riordan arrays.

Theorem 6. Let $n$ be nonegative integers, $k, r, p \geq 1$ are integers, we have

$$
\sum_{m=0}^{n} C h_{m}^{(k)}(\alpha x+p) \beta^{m} n !(-1)^{n-m+r} H_{n-m, p, r}(\alpha, \beta)=C G_{n}^{k, r}(\alpha, \beta \mid x) .
$$




\section{Proof}

$$
\begin{aligned}
\sum_{n=0}^{\infty} C G_{n}^{k, r}(\alpha, \beta \mid x) \frac{t^{n}}{n !} & =\left(\frac{2}{2+\beta t}\right)^{k}(1+\beta t)^{(\alpha x+p)} \frac{\log ^{r}(1+\alpha t)}{(1+\beta t)^{p}} \\
& =\sum_{n=0}^{\infty} C h_{n}^{(k)}(\alpha x+p) \beta^{n} \frac{t^{n}}{n !} \sum_{n=0}^{\infty}(-1)^{n+r} H_{n, p, r}(\alpha, \beta) t^{n} \\
& =\sum_{n=0}^{\infty} \sum_{m=0}^{n} C h_{m}^{(k)}(\alpha x+p) \beta^{m}(-1)^{n-m+r} H_{n-m, p, r}(\alpha, \beta) \frac{t^{n}}{m !} .
\end{aligned}
$$

By comparing the coefficients of $t^{n}$ on both sides of this equation, theorem 6 is proved.

Corollary 3. In theorem 6 , when $\alpha=\beta$, and $p=1$, the following relations hold:

$$
\sum_{m=0}^{n}\left(\begin{array}{l}
n \\
m
\end{array}\right) \frac{\alpha^{n}(-1)^{n-m+r}}{(n-m) !} C h_{m}^{(k)}(\alpha x+1) H(n-m, r-1)=C G_{n}^{k, r}(\alpha, \alpha \mid x) .
$$

Theorem 7. Let $n \geq r, k \geq 1$ and $r \geq 1$ are integers, then

$$
\sum_{m=0}^{n-r}\left(\begin{array}{c}
n-r \\
m
\end{array}\right) C h_{m}^{(k)}(\alpha x) \beta^{m} \alpha^{r} D_{n-r-m, \alpha}^{(r)} \frac{n !}{(n-r) !}=C G_{n}^{k, r}(\alpha, \beta \mid x) .
$$

Proof According to the equation (4) and (5), we have

$$
\begin{aligned}
\sum_{n=0}^{\infty} C G_{n}^{k, r}(\alpha, \beta \mid x) \frac{t^{n}}{n !} & =\frac{2^{k} \log ^{r}(1+\alpha t)}{(2+\beta t)^{k}}(1+\beta t)^{\alpha x} \\
& =\left(\frac{2}{2+\beta t}\right)^{k}(1+\beta t)^{\alpha x} \frac{\log ^{r}(1+\alpha t)}{(\alpha t)^{r}}(\alpha t)^{r} \\
& =\sum_{n=0}^{\infty} C h_{n}^{(k)}(\alpha x) \frac{(\beta t)^{n}}{n !} \sum_{n=0}^{\infty} D_{n, \alpha}^{(r)} \frac{t^{n}}{n !}(\alpha t)^{r} \\
& =\sum_{n=0}^{\infty} \sum_{m=0}^{n}\left(\begin{array}{c}
n \\
m
\end{array}\right) C h_{m}^{(k)}(\alpha x) \beta^{m} \alpha^{r} D_{n-m, \alpha}^{(r)} \frac{\alpha^{r} t^{n+r}}{n !} .
\end{aligned}
$$

By comparing the coefficients of $t^{n}$ on both sides of this equation, theorem 7 is proved.

Corollary 4. In theorem 7 , when $\alpha=1$, the following relation holds:

$$
\sum_{m=0}^{n-r}\left(\begin{array}{c}
n-r \\
m
\end{array}\right) C h_{m}^{(k)}(x) \beta^{m} D_{n-r-m}^{(r)} \frac{n !}{(n-r) !}=C G_{n}^{k, r}(1, \beta \mid x) .
$$

Corollary 5. In theorem 7 , when $\alpha=\beta=1$, and $k=r$, the following relation holds:

$$
\sum_{m=0}^{n-r}\left(\begin{array}{c}
n-r \\
m
\end{array}\right) C h_{m}^{(r)}(x) D_{n-r-m}^{(r)} \frac{n !}{(n-r) !}=C G_{n}^{(r)}(x) .
$$

Theorem 8. Let $n \geq \min \{r, s\}, k, r, s \geq 1$ are integers, we have

$$
\sum_{m=0}^{n} \alpha^{n-m} b_{n-m}^{(s)} \frac{C G_{m}^{k, r}(\alpha, \beta \mid x)}{m !(n-m) !}= \begin{cases}C G_{n-s}^{k, r-s}(\alpha, \beta \mid x) \frac{\alpha^{s}}{(n-s) !}, & r>s \\
C h_{n-s}^{(k)}(\alpha x) \alpha^{s} \beta^{n-s} \frac{1}{(n-s) !}, & r=s \\
\sum_{m=0}^{n-r}\left(\begin{array}{c}
n-r \\
m
\end{array}\right) \frac{\beta^{m} \alpha^{n-m}}{(n-r) !} C h_{m}^{(k)}(\alpha x) b_{n-r-m}^{(s-r)} . & r<s\end{cases}
$$


Proof By equation (8),(11),(13), we have

$$
\begin{aligned}
& \sum_{m=0}^{n} b_{n-m}^{(s)} \frac{C G_{m}^{k, r}(\alpha, \beta \mid x)}{\left(\begin{array}{c}
n \\
m
\end{array}\right)^{-1} \alpha^{m-n} n !}=\left[t^{n}\right] \alpha^{s} t^{s} \frac{2^{k} \log ^{r-s}(1+\alpha t)}{(2+\beta t)^{k}} \\
& = \begin{cases}C G_{n-s}^{k, r-s}(\alpha, \beta) \frac{\alpha^{s}}{(n-s) !}, & r>s \\
C h_{n-s}^{(k)}(\alpha x) \alpha^{s} \beta^{n-s} \frac{1}{(n-s) !}, & r=s \\
\sum_{m=0}^{n-r}\left(\begin{array}{c}
n-r \\
m
\end{array}\right) \frac{\beta^{m} \alpha^{n-m}}{(n-r) !} C h_{m}^{(k)}(\alpha x) b_{n-r-m}^{(s-r)} . & r<s\end{cases}
\end{aligned}
$$

Theorem 9. Let $k, r \geq 1$ and $p, l \geq 0$ be integers, we have

$$
\sum_{j=0}^{r} \sum_{p=0}^{j} \sum_{l=0}^{r-j}\left(\begin{array}{l}
r \\
j
\end{array}\right)\left(\begin{array}{l}
k \\
l
\end{array}\right) \beta^{l} \frac{s_{2}(j, p) s_{2}(r-j, l)}{2^{l} \alpha^{p+l}} C G_{p}^{k, r}(\alpha, \beta) \frac{l !}{r !}=\delta_{n, r},
$$

where $\delta_{n, r}$ is the Kronecker delta symbol.

Proof Replacing $t$ by $\frac{e^{t}-1}{\alpha}$ in equation (13), we have

Hence, we have

$$
\sum_{n=0}^{\infty} C G_{n}^{k, r}(\alpha, \beta) \alpha^{-n} \frac{\left(e^{t}-1\right)^{n}}{n !}=\frac{2^{k} t^{r}}{\left[2+\frac{\beta\left(e^{t}-1\right)}{\alpha}\right]^{k}} .
$$

$$
\sum_{n=0}^{\infty} C G_{n}^{k, r}(\alpha, \beta) \alpha^{-n} \frac{\left(e^{t}-1\right)^{n}}{n !}\left[2+\frac{\beta\left(e^{t}-1\right)}{\alpha}\right]^{k}=2^{k} t^{r} .
$$

As is well known, $\sum_{n=k}^{\infty} s_{2}(n, k) \frac{t^{n}}{n !}=\frac{\left(e^{t}-1\right)^{k}}{k !}(\operatorname{see}[12])$.

Now, we consider the left-hand side of the equation (29), we have

$$
\begin{aligned}
& \sum_{n=0}^{\infty} C G_{n}^{k, r}(\alpha, \beta) \alpha^{-n} \frac{\left(e^{t}-1\right)^{n}}{n !}\left[2+\frac{\beta\left(e^{t}-1\right)}{\alpha}\right]^{k} \\
& =\sum_{m=0}^{\infty} \sum_{n=0}^{m} C G_{n}^{k, r}(\alpha, \beta) \alpha^{-n} s_{2}(m, n) \frac{t^{m}}{m !} \sum_{i=0}^{\infty} \sum_{l=0}^{i}\left(\begin{array}{l}
k \\
l
\end{array}\right) 2^{k-l}\left(\frac{\beta}{\alpha}\right)^{l} l ! s_{2}(i, l) \frac{t^{i}}{i !} \\
& =\sum_{n=0}^{\infty} \sum_{j=0}^{n} \sum_{p=0}^{j} \sum_{l=0}^{n-j}\left(\begin{array}{l}
n \\
j
\end{array}\right)\left(\begin{array}{l}
k \\
l
\end{array}\right) \beta^{l} \frac{s_{2}(j, p) s_{2}(n-j, l)}{2^{l-k} \alpha^{p+l}} C G_{p}^{k, r}(\alpha, \beta) \frac{l !}{n !} t^{n} .
\end{aligned}
$$

By comparing the coefficients of $t^{r}$, theorem 9 is proved.

By the concept of Riordan arrays and equation (13), we get $\left\{\frac{C G_{n}^{k, r}(\alpha, \beta)}{n !}\right\}=\left(2^{k}(2+\beta t)^{-k}, \ln (1+\alpha t)\right)$, we can get the follwing results:

Theorem 10. Let $n, k \geq 1$ be integers, we have

$$
\sum_{j=1}^{n} C G_{n}^{k, j}(\alpha, \beta) \frac{1}{j !}=n \alpha \beta^{n-1} C h_{n-1}^{(k)} .
$$

\section{Proof}

$$
\begin{aligned}
& \sum_{j=1}^{n} C G_{n}^{k, j}(\alpha, \beta) \frac{1}{n !} \frac{1}{j !}=\left[t^{n}\right] 2^{k}(2+\beta t)^{-k}\left[e^{y}-\left.1\right|_{y=\log (1+\alpha t)}\right] \\
& =\left[t^{n-1}\right] 2^{k} \alpha(2+\beta t)^{-k}=\left[t^{n-1}\right] \alpha \sum_{n=0}^{\infty} C h_{n}^{(k)} \beta^{n} \frac{t^{n}}{n !} \\
& =\alpha \beta^{n-1} \frac{C h_{n-1}^{(k)}}{(n-1) !} .
\end{aligned}
$$

Hence, the identity (30) can be obtained immediately. 
Theorem 11. Let $n, k, j, m \geq 1$ be integers, we set up the following equation:

$$
\begin{aligned}
& \sum_{j=1}^{n} C G_{n}^{k, j}(\alpha, \beta) \frac{G_{j}^{(m)}}{n ! j !}=\sum_{l=0}^{n}\left(\begin{array}{c}
n-l+m-1 \\
m-1
\end{array}\right) \frac{(-\alpha)^{n-l}}{2^{n-l} l !} C G_{l}^{k, m}(\alpha, \beta), \\
& \sum_{j=1}^{n} C G_{n}^{k, j}(\alpha, \beta) \frac{G_{j}^{(x)}}{2^{j} n ! j !}=\sum_{l=0}^{n}\left(\begin{array}{c}
l+k-1 \\
k-1
\end{array}\right)\left(\begin{array}{c}
-x \\
n-l
\end{array}\right)\left(-\frac{1}{2}\right)^{n} \alpha^{n-l} \beta^{l} .
\end{aligned}
$$

Proof

$$
\begin{aligned}
& \sum_{j=1}^{n} C G_{n}^{k, j}(\alpha, \beta) \frac{1}{n !} \frac{G_{j}^{(m)}}{j !}=\left[t^{n}\right] 2^{k}(2+\alpha t)^{-k}\left[\left.\left(\frac{2 y}{e^{y}+1}\right)^{m}\right|_{y=\log (1+\alpha t)}\right] \\
& =\left[t^{n}\right] \frac{2^{k} \log ^{m}(1+\alpha t)}{(2+\beta t)^{k}} \frac{2^{m}}{(2+\alpha t)^{m}}=\left[t^{n}\right] \sum_{n=0}^{\infty} C G_{n}^{k, m}(\alpha, \beta) \frac{t^{n}}{n !} \sum_{n=0}^{\infty}\left(\begin{array}{c}
n+m-1 \\
m-1
\end{array}\right)\left(-\frac{\alpha}{2}\right)^{n} t^{n} \\
& =\sum_{l=0}^{n}\left(\begin{array}{c}
n-l+m-1 \\
m-1
\end{array}\right) \frac{(-\alpha)^{n-l}}{2^{n-l} l !} C G_{l}^{k, m}(\alpha, \beta) .
\end{aligned}
$$

Hence, the equation (31) is proved. The proof of (32) is similar to that of (31), and it is omitted here.

Corollary 6. In theorem 11 , when $\alpha=\beta$, the following relations hold:

$$
\begin{aligned}
& \sum_{j=1}^{n} C G_{n}^{k, j}(\alpha, \alpha) \frac{G_{j}^{(m)}}{j !}=C G_{n}^{k+m, m}(\alpha, \alpha) \\
& \sum_{j=1}^{n} C G_{n}^{k, j}(\alpha, \beta) \frac{G_{j}^{(x)}}{2^{j} n ! j !}=\left(-\frac{\alpha}{2}\right)^{n}\left(\begin{array}{c}
n+k+x-1 \\
n
\end{array}\right) .
\end{aligned}
$$

Theorem 12. Let $n, k, j, m \geq 1$ be integers, we set the following equations:

$$
\sum_{j=1}^{n} C G_{n}^{k, j}(\alpha, \beta)\left(\begin{array}{c}
n+m \\
n
\end{array}\right) \frac{m ! B_{j}^{(m)}}{j !}=\alpha^{-m} C G_{n+m}^{k, m}(\alpha, \beta) .
$$

\section{Proof}

$$
\begin{aligned}
& \sum_{j=1}^{n} C G_{n}^{k, j}(\alpha, \beta) \frac{1}{n !} \frac{B_{j}^{(m)}}{j !}=\left[t^{n}\right] 2^{k}(2+\alpha t)^{-k}\left[\left.\left(\frac{y}{e^{y}-1}\right)^{m}\right|_{y=\log (1+\alpha t)}\right] \\
& =\left[t^{n}\right] \frac{2^{k} \log ^{m}(1+\alpha t)}{(2+\beta t)^{k}}(\alpha t)^{-m}=C G_{n+m}^{k, m}(\alpha, \beta) \frac{\alpha^{-m}}{(n+m) !} .
\end{aligned}
$$

Hence, theoerm 12 can be obtained immediately.

The second and third sections are our main results. We generalize the generating function of the Changhee-Genocchi polynomials and find some new identities by the method of generating functions and Riordan arrays. Specially, these identities contain some relations about classical Changhee-Genocchi polynomials. In addition, it is easy to see that combinations of special sequences can be represented by the generalized Changhee-Genocchi polynomials, such as the Changhee polynomials and the generalized Harmonic numbers, the Changhee polynomials and the Daehee numbers, etc.

Acknowledgements. The research is supported by the Natural Science Foundation of China under Grant 11461050 and Natural Science Foundation of Inner Mongolia under Grant 2016MS0104. 


\section{References}

1. B. M. Kim, J. Jeong, and S. H. Rim, "Some explicit identities on changhee-genocchi polynomials and numbers," Adv. Differ. equ., vol. 2016, no. 1, p. 202, 2016.

2. F. Z. Zhao and Wuyungaowa, "Some results on a class of generalized harmonic numbers," Utilitas Mathematica, vol. 87, no. 1, pp. 65-87, 2012.

3. D. S. Kim and T. Kim, "Higher-order changhee numbers and polynomials," Adv. Studies Theor. Phys., vol. 8, no. 8, pp. 365-373, 2014.

4. D. S. Kim, T. Kim, J. J. Seo, and S. H. Lee, "A note on the twisted lambda-daehee polynomials," Applied Mathmetical Sciences, vol. 7, no. 141, pp. 7005-7014, 2013.

5. G. D. Liu, "Arithmetic identities involving genocchi and stirling numbers," Discrete Dynamics in Nature and Society, vol. 2009, no. 1, pp. 332-337, 2014.

6. L. Carlitz, "A note on bernoulli and euler polynomials of the second kind," Scripta Math, vol. 25, pp. 323-330, 1961.

7. G. D. Liu and H. M. Srivastava, "Explicit formulas for the nörlund polynomials of the first and second kind," Computers and Mathematics with Applicatios, vol. 51, no. 9-10, pp. 1377-1384, 2006.

8. D. S. Kim and T. Kim, "Some identities of higher order euler polynomials arising from euler basis," Integral Transforms and Special Functions, vol. 24, no. 9, pp. 734-738, 2013.

9. D. S. Kim, T. Kim, and D. V. Dolgy, "Bernoulli polynomials of the second kind and their identities arising from umbral calculus," J. Nonlinear Sci. Appl., vol. 9, no. 3, pp. 860-869, 2016.

10. R. Sprugnoli, "Riordan arrays and combinatorial numbers," Discrete Math, vol. 132, pp. 267-290, 1994.

11. F. Qi, "A recurrence formula for the first kind stirling numbers," Eprint Arxiv., vol. 10, 2013.

12. B. N. Guo and F. Qi, "An explicit formula for bernoulli numbers in terms of stirling numbers of the second kind," J. Ana. Num. Theor., vol. 3, no. 1, pp. 27-30, 2015. 